\title{
Optical Design of the WFIRST Phase-A Wide Field Instrument
}

\author{
Bert A Pasquale ${ }^{\mathrm{a}}$, Catherine T Marx ${ }^{\mathrm{a}}$, Guangjun Gao ${ }^{\mathrm{b}}$, Nerses Armani $^{\mathrm{c}}$, Thomas Casey ${ }^{\mathrm{d}}$ \\ ${ }^{a}$ NASA/Goddard Space Flight Center, Greenbelt, MD 20771, ' Sigma Space Inc. 4600 Forbes Blvd,

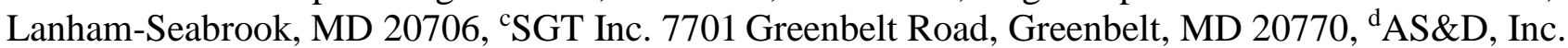 \\ 7000 Muirkirk Meadows Dr., Beltsville, Maryland 20705 \\ Bert.Pasquale@nasa.gov
}

\begin{abstract}
The WFIRST Wide-Field Infrared Survey Telescope TMA optical design provides 0.28 -sq ${ }^{\circ}$ FOV at 0.11 " pixel scale to

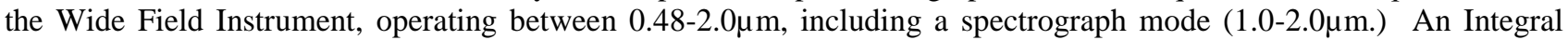
Field Channel provides 2-D discrete spectroscopy at 0.15 " \& 0.3 " sampling.
\end{abstract}

Keywords: Space Instrumentation, Astronomical Optics, Imaging Systems, Telescopes, Dark Energy, Spectroscopy

\section{INTRODUCTION}

The optical design of the Wide-Field Infra-Red Survey Telescope (WFIRST) ${ }^{[1]}$ Wide-Field Channel (WFC) provides $\sim 1 / 3$-square degree of instantaneous field coverage at 0.11 arcsecond pixel scale. This is accomplished using a 2.4-meter aperture Three Mirror Anastigmat (TMA) optical design that was constrained and influenced by a combination of existing hardware, design heritage, science interests and architecture constraints. The result is a design with diffractionlimited imaging over a focal plane of 300.8 million pixels, operating in seven panchromatic bands between $0.48-$ $2.0 \mu \mathrm{m}$, or multi-spectral imaging mode from 1.0-2.0 $\mu \mathrm{m}$. Within the instrument enclosure is a separate Integral Field Channel (IFC) providing discrete spectral analysis over 3"x4.5" and 4.2"x9" fields $(0.15$ " and 0.3 " sampling, respectively) and $\mathrm{R}=100$ spectral resolution.

\section{OVERVIEW OF WFIRST AND THE WIDE FIELD CHANNEL}

This incarnation of WFIRST's optical design is designated as the Preliminary Phase-A design (v 7.6.8), and will be the

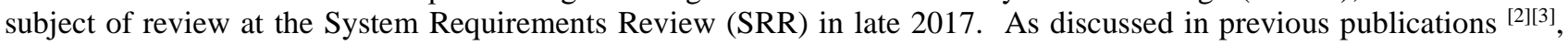
the observatory's Imaging Optics Assembly (IOA) uses a repurposed 2.4-m Forward Optical Assembly (FOA) donated from the intelligence community, repurposed by NASA for scientific use. (See Figure 1.)

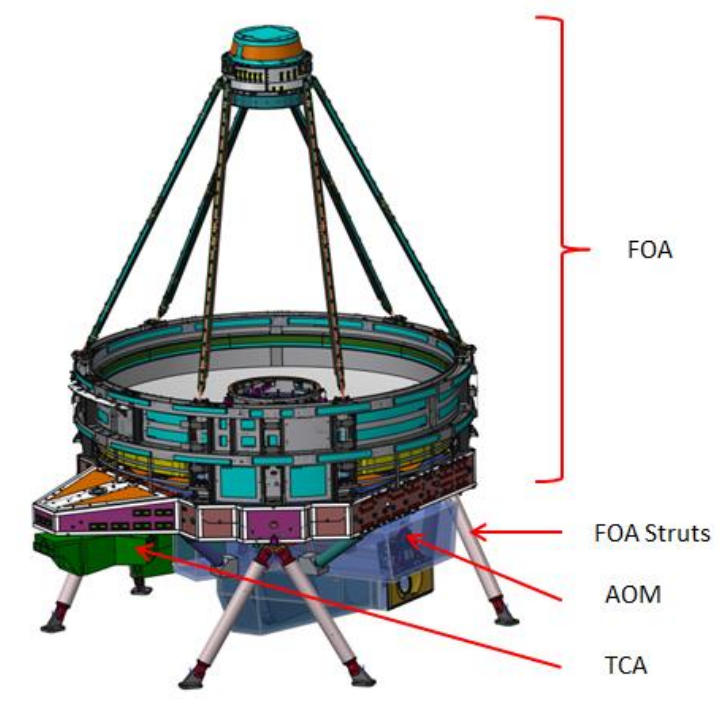

Figure 1(a). The components of the WFIRST Imaging Optics Assembly (IOA) built around the heritage FOA. 
WFIRST has the advantage (and challenge) of designing around existing hardware. Since beginning the design cycles in 2012, the project team has conducted many tests of the existing hardware and materials. To assure our understanding of the hardware is complete, the team continues to conducted inspections of the telescope components. The two existing optical components of the FOA are the Primary Mirror (PM) and Secondary Mirror (SM). (See figures 2 (a) \& (b).) They are supported off of the Aft Metering Structure (AMS). To complete the IOA, an Aft Optics Module (AOM) containing the WFC Aft Optics (WAO) and IFC Relay Optics (IRO) hangs under the AMS. Also attached to the AMS is the CGI Tertiary Mirror Assembly (TCA). The IOA is supported on the spacecraft bus via. the FOA struts.
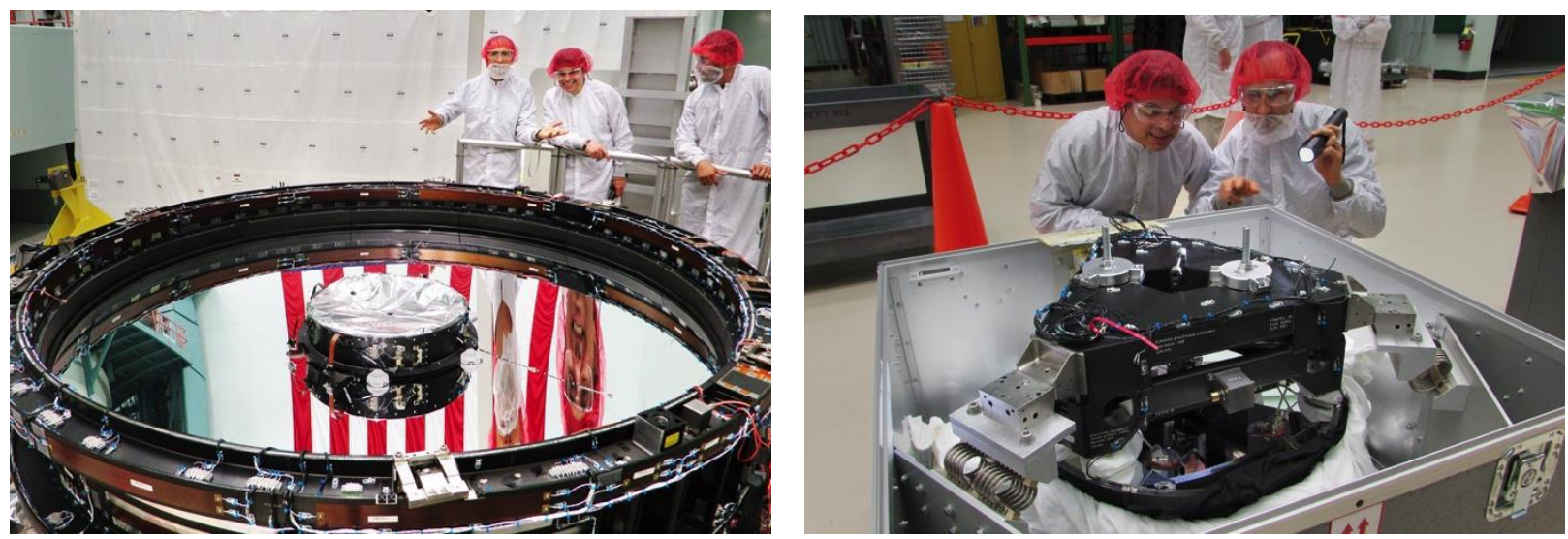

Figure 2(a). The author with the WFIRST Primary mirror during a 2016 hardware inspection. (Left to right: Bert Pasquale, Optical Designer, Nerses Armani Mechanical designer, and Tom ) (b) The B. Pasquale and N. Armani with the WFIRST Secondary Mirror Assembly. (The mirror can be seen under the assembly from the back.) Images are ITAR-cleared.

WFIRST carries both the Wide Field Instrument (WFI) and the Coronagraph Instrument (CGI). An Instrument Carrier (IC) meters these payload elements together and to the spacecraft bus. However the design has undergone a major renovation since the Mission Concept Review (MCR) design of December 2015. These changes will be discussed in detail in the following sections.

\section{SCIENTIFIC SIGNIFICANCE}

The Wide-Field Infrared Survey Telescope (WFIRST) mission is the top-ranked large space mission in the New Worlds, New Horizon (NWNH) Decadal Survey of Astronomy and Astrophysics. WFIRST is conceived to settle essential questions in both exoplanet and dark energy research and will advance topics ranging from galaxy evolution to the study of objects within the galaxy. As such, WFIRST's Wide Field Instrument was designed to tackle two key questions about the acceleration of the universe. ${ }^{[4]}$ First, it will address whether the speed-up is caused by a new "dark energy" or simply by the breakdown of our understanding of gravity on cosmological scales. Second, if the acceleration is due to a new energy component, WFIRST will discern if its energy density is constant in space and time or whether it has evolved over the history of the universe. To achieve this, the observatory will carry out three types of surveys. (See Figure 3.)

First, an imaging survey will measure the shapes and redshifts of a large number of 350 million galaxies and 40,000 galaxy clusters, and will observe how their images are stretched by "gravitational weak lensing" as they pass tangentially around the location of dark matter. The results will produce a map of dark matter and cosmic structure rate of growth, thereby tracing out the distribution and time-evolution of dark energy. Second, WFIRST will carry out a spectral survey of 22 million galaxies to study the imprint of primordial sound waves on the clustering of galaxies, also known as baryon acoustic oscillations, providing an absolute calibration of the expansion rate history, as well as tests of general relativity based on localized redshift distortions in galaxy clusters. In addition to these two "wide-field" surveys, a third supernova survey will observe the spectral evolution of 2,700 type 1a supernovae via integral-field spectroscopy to correlate their distance (inferred by their brightness) and speed (inferred from their redshift).

Taken together, these three surveys vastly increase the accuracy of the 3D maps of the distribution of matter and gravity, and measure the expansion history of the universe with an aggregate precision of $0.1-0.5 \%$. This will be improved further as WFIRST's cosmological data is combined with ground-based instruments such as the Large Synoptic Survey Telescope and other space-based missions such as ESA's Euclid telescope. 


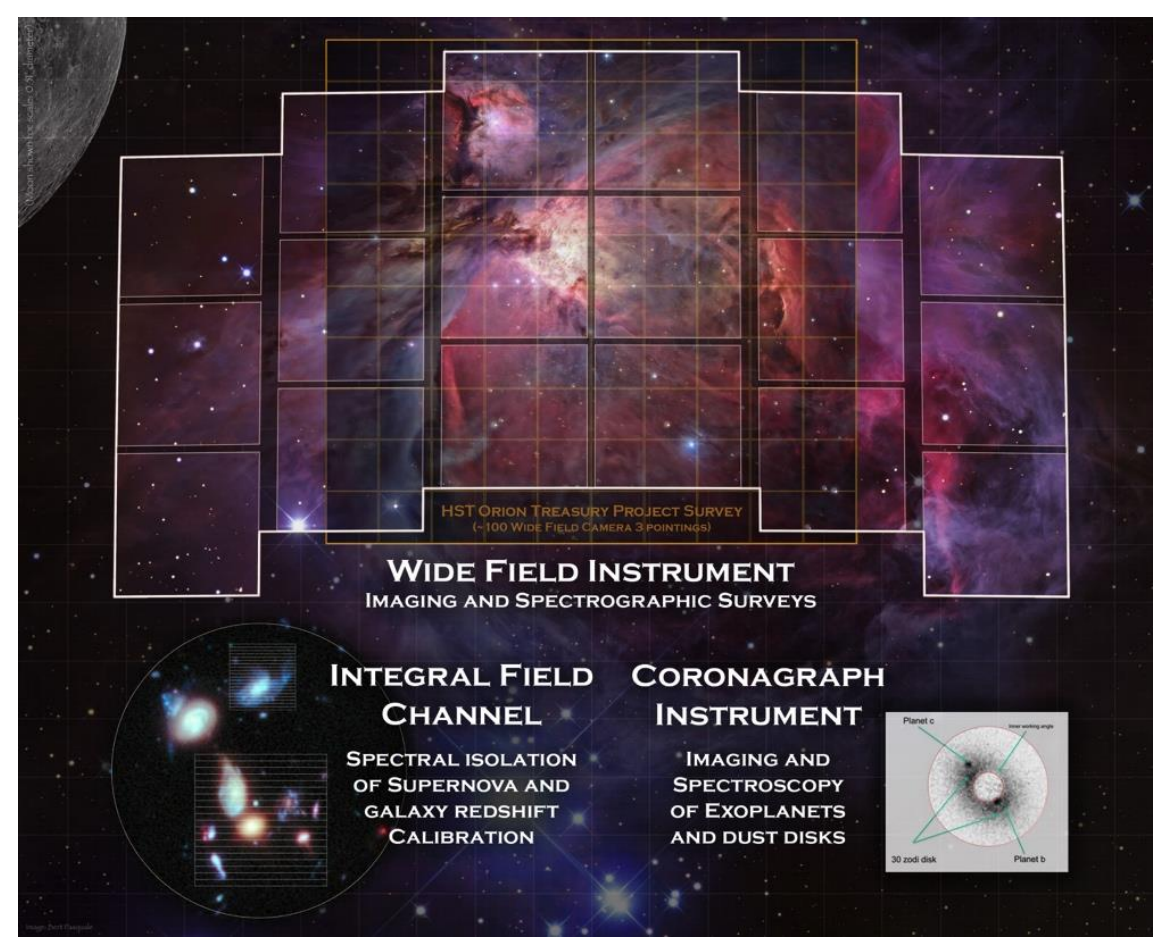

Figure 3. WFIRST's WFI Imaging and Spectral Survey Field of View covers a moon-sized area (shown for scale) of the sky with 300 megapixels, which is 90-200 times larger than the cameras in the Hubble or James Webb space telescopes. The IFC dissects supernova spectrums from the distant host galaxy, and the CGI observes exoplanetary systems between 0.1 to 1 arcsecond from the host star (simulated image shown.)

In addition to the wide-field surveys, WFIRST will also host a demonstration of technology for the first space borne wavefront-controlled exoplanet Coronagraph Instrument. The CGI has potential to image dozens of planetary systems orbiting other stars within 10 parsecs. The CGI will be able to see reflected light from both far-orbiting mature gas giants and smaller, highly reflective rocky planets nearer to their host star, as well as map the structure of exo-zodiacal dust disks. Direct imaging will even make it possible to detect changes in both brightness and spectrum as some exoplanets rotate on their axes, indicating differences in land coverage.

\section{OBSERVATORY AND PHASE-A ARCHITECTURE CHANGES}

The previous MCR design had the disadvantage of being a "divided" telescope; the tertiary mirror was mounted within the WFI assembly, apart from the primary and secondary structure. This made verifying the requirements for the both Wide Field Instrument and the telescope more difficult. Because the instrument interface was at the caustic intermediate image, a series of steps involving holographic optical elements and translation stages were envisioned. While these I\&T and wavefront sensing methods are in themselves robust, they accounted for several additional terms to the error budget. In the new architecture, the entire TMA is delivered with imaging specifications directly tied to scientific performance. GSE still includes interferometers and wavefront sensing, but is done on the final exit pupil and image that will be projected to the focal plane. I\&T and stability error budgets are improved all around with a unified structure.

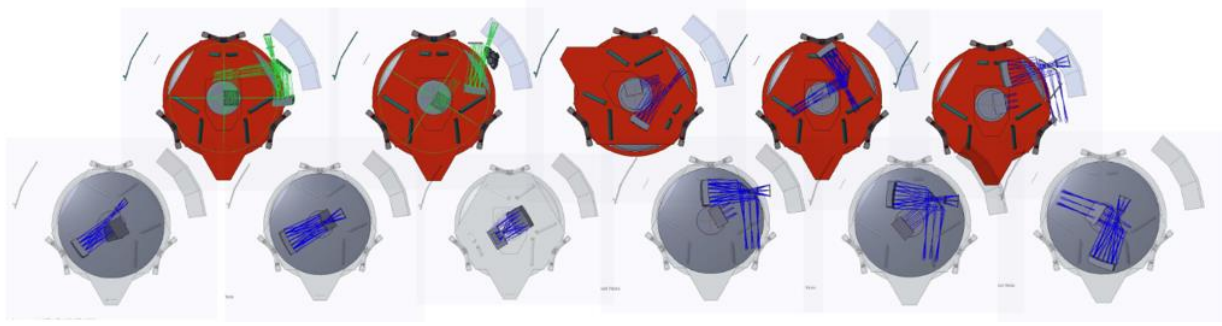

Figure 4. Several of the WFIRST Architectures that were considered as part of the trade study. 
Once it was decided to move the Tertiary Mirror to a dedicated Aft Optics Module in the telescope, a flurry of activity ensued. One "tiger team" looked at thermal zones and passive radiative cooling, which has an influence on focal plane placement requirements. The structures, optics, and systems engineers conferred heavily with the telescope vendor on how the various options could play out. With three instruments vying for space and needing to satisfy their respective constraints, 20 optics layouts were considered, some of which are shown in Figure 4, above. The aft optics were either folded to traverse under the metering structure, stayed in a single telescope-WFC meridian plane, or used a meridiantransverse hybrid fold. Both the exit pupil and the focal plane needed to be accessible and not buried within the structure. It was only after an intense matrix study of these options that a 21 st layout was considered that best balanced symmetry, existing hardware constraints, clearances and connections to other instruments, pupil accessibility and focal plane placement. This became the preliminary Phase-A baseline architecture, which is described in this paper and shown below in Figure 5.

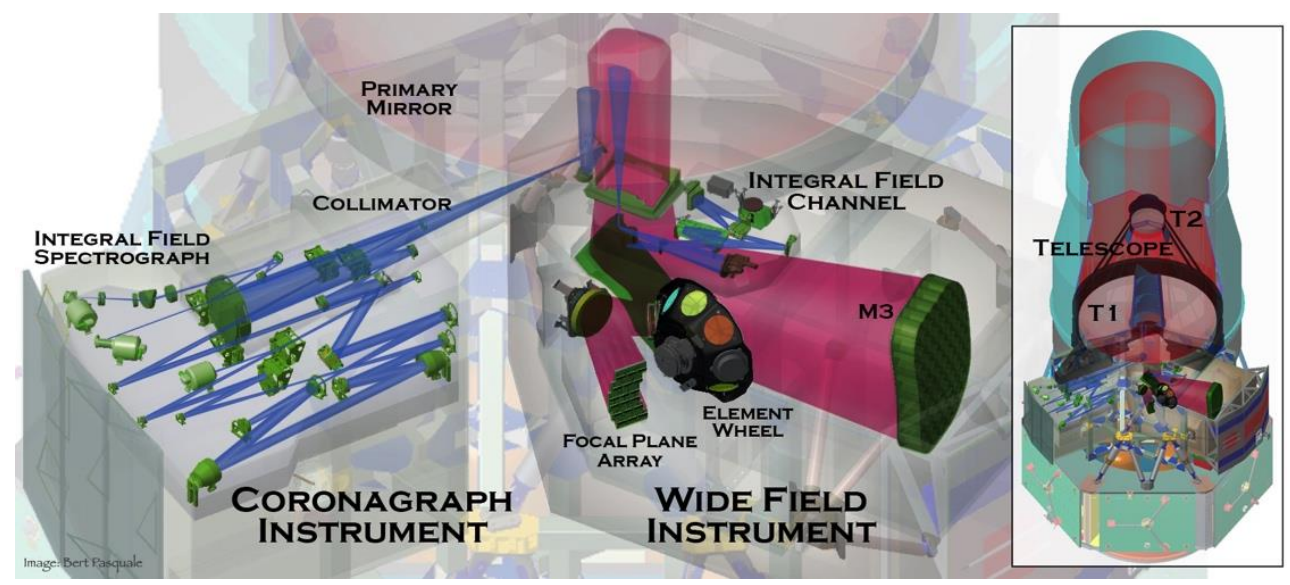

Figure 5. The WFIRST Phase-A Observatory showing the telescope, Wide Field Instrument (WFI) and Coronagraph Instrument (CGI.)

In Figure 6 (below), you can see how the back focal length of the FOA was increased to allow placement of the focal plane towards the radiator. The intermediate focus Entrance Aperture Plate (EAP) is now located between the two WFC Aft Optics fold mirrors. This then allows the Tertiary Mirror to hang evenly below the telescope, and very near the observatory centerline. The exit pupil is projected out beyond Fold Mirror 2, freeing it from the constraint of being wrapped inside of the passing ray bundle. This allows the connection between the telescope and Wide Field Instrument a clean interface using a light and thermal labyrinth seal.

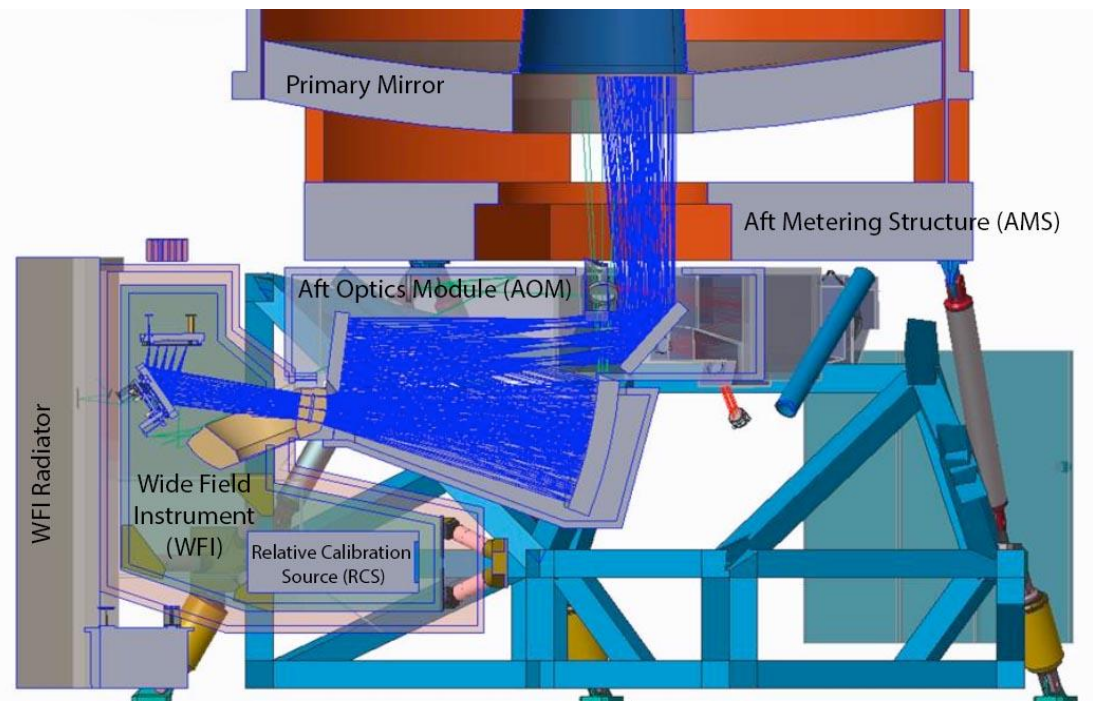

Figure 6. The WFIRST Phase-A Observatory Meridian plane cut-through of the Wide Field Channel. 
The WFC design also includes the Integral Field Channel Relay System, and accommodates the volume constraints of the CGI pickoff and Tertiary Collimator Assembly.

\section{WIDE FIELD CHANNEL (WFC) OPTICAL LAYOUT}

\subsection{Aft Optics Module (AOM)}

The WFC ray bundles both enter into the Aft Optics Module prior to the intermediate "Cassegrain" focus. Once inside, the first fold mirror (FM1) acts as a pickoff for an off-axis section of the field, where the Entrance Aperture Plate (EAP) acts as a field limiter and stray light baffle for the WFC. A second fold mirror (FM2) folds back towards the axis, where the only powered mirror within the WAO, the Tertiary Mirror (TM) works in concert with the PM \& SM to produce the corrected WFC image. All three powered mirrors of the TMA are simple conics.

By minimizing the distance from FM2 to the TM, the TMA exit pupil is able to be projected outside of the AOM. Once the ray bundle passes the telescope exit pupil, it enters into the Wide field instrument. The WFI WFC optics consists of a mechanized Element Wheel, focusing element, and Focal Plane Array (FPA). The Instrument Fold Mirror provided focus adjustment during I\&T and on-orbit. The WFC optical layout is shown in Figure 7.

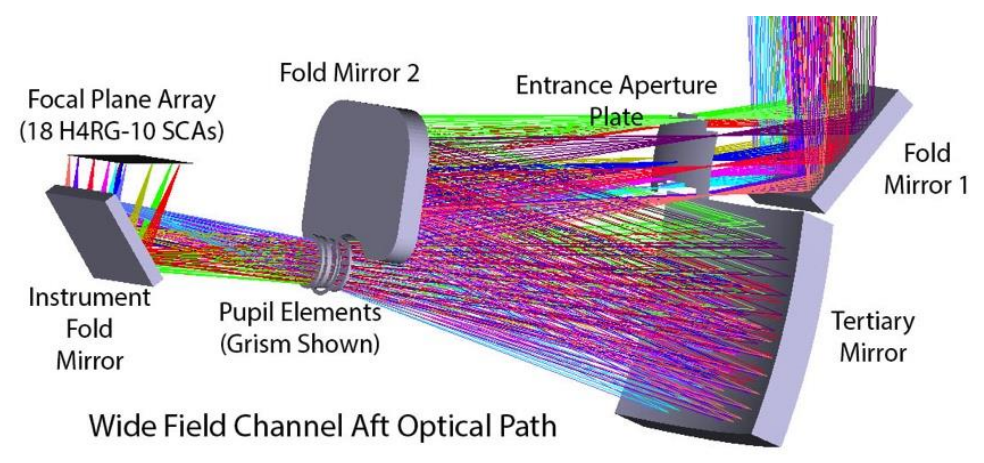

Figure 7. Optical Layout of the WFIRST (Phase-A SRR Design) Wide-Field Channel

\subsection{Element Wheel}

Inside the WFI, the beam first passes through the element wheel optical elements. The new architecture has allowed the element wheel to have increased capacity to 10 positions, currently slated for six $R=4$ bandpass filters, one wide filter and one multi-element grism assembly. Figure 8a shows two occupied positions. The filters are very weak meniscus lenses designed to maintain par-focality when placed in the WFC beam. Longwave filters have an integrated Lyot Stop.
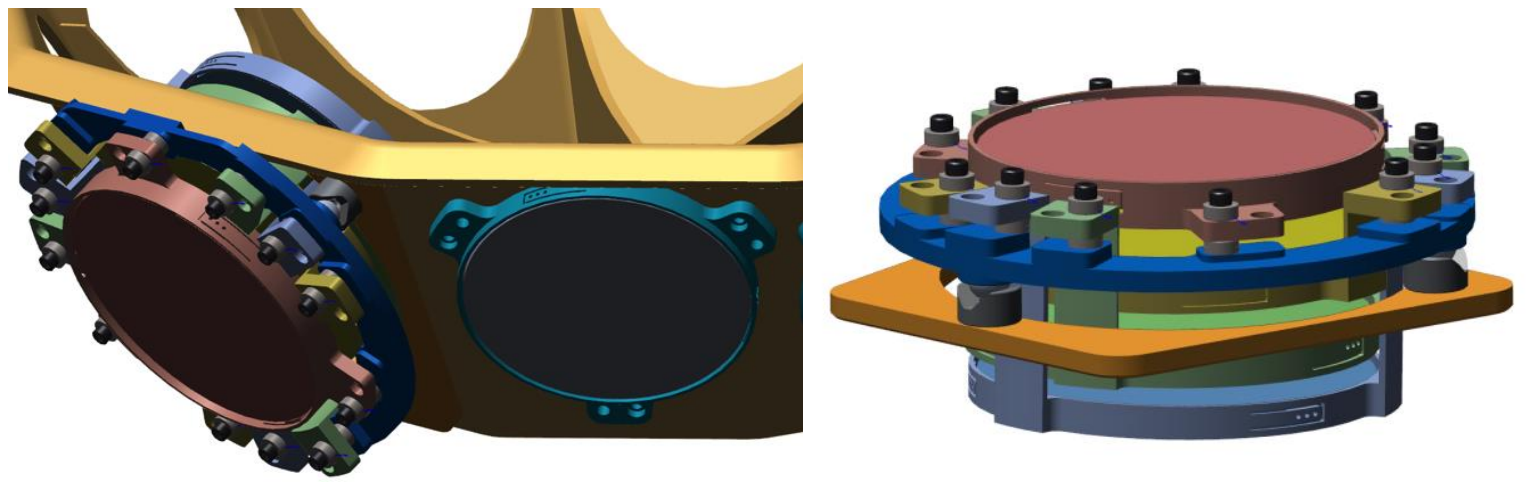

Figure 8 (a) WFC Element Wheel showing the Grism and a bandpass filter. (b) A breakout model of the Grism Assembly

The grism consists of 4 optical elements E1 - 4 (Figure 8b), with an integrated Lyot Stop. E1 \& E4 are plano binary diffractive elements which balance dispersion and aberrations. E2 \& E3 are powered prism elements with spherical-only surfaces. E1 mounts into the baseplate, then E2-4 in their respective mounts are successively assembled to the plate, each with adjustable positioning via spherical washers that can provide 6-DOF on each element. 


\subsection{Instrument Fold Mirror (IFM) \& Focus Mechanism}

The Instrument Fold Mirror (IFM) prior to the focal plane folds the final image towards the observatory radiator interface. With the FPA mounted in place, the IFM alignment mechanism provides 3-DOF (tip/Tilt/focus) adjustment to bring the WFC image into alignment across the full focal plane.

\subsection{WFC Focal Plane Array (FPA)}

At the Prime focus of the WFIRST TMA Telescope is the imaging array for the Wide Field Channel. The Focal Plane Array carries 18 individual H4RG-10 detectors, each with 4,088x4,088 $10 \mu \mathrm{m}$ active pixels (16.7 Mpix). Each sensor is co-planar allowing for simplified assembly and testing. The sensors are laid out in a 6 columns of 3 sensors each (as seen in Figure 9a.) An integrated light shield on each SCA has a window custom cut for that SCA's light bundle acceptance angle (see Figure 9b.)
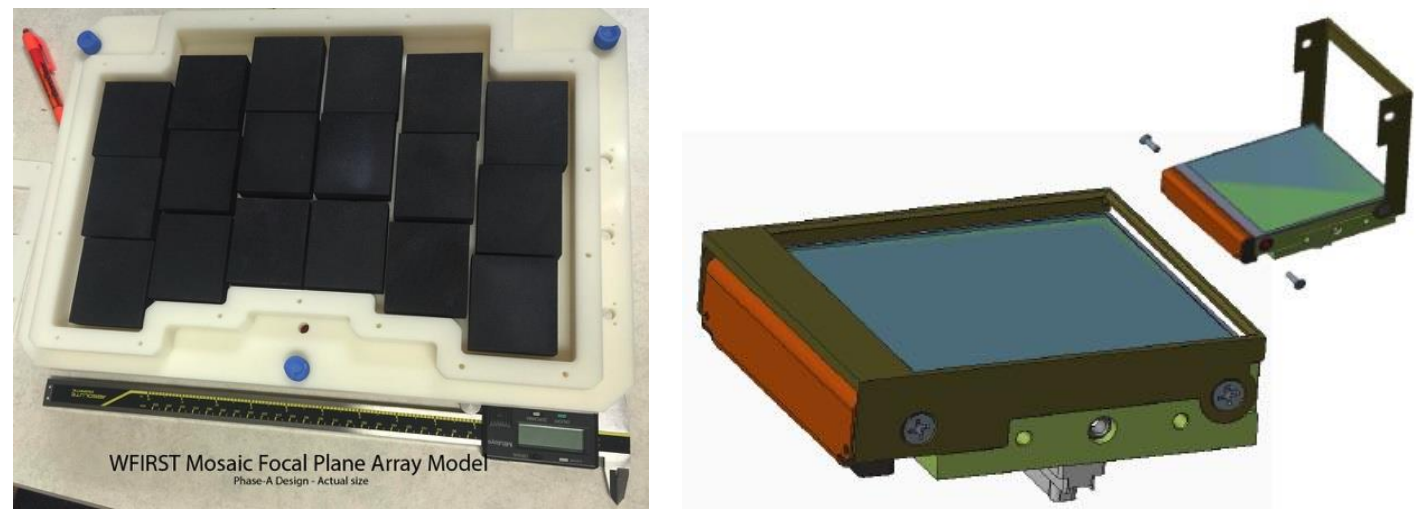

Figure 9. (a) 3-D printed model of an early Phase-A FPA and 18 H4RG SCAs. (b) SCA model with integrated light shield.

The flight FPA is not installed until final integration of the WFI. The telescope, instruments, and assembled payload will each go through thermal vacuum tests and gravity modeling. These are further verified by various subsystem tests. Once assembled, the payload will experience a full double pass optical verification test using an Auto-Collimating Mirror (ACM.) The sources for this test are fibers embedded in the FPA, adjacent to the active pixel areas.

\section{WIDEFIELD IMAGER MODE (WIM) PERFORMANCE}

By offsetting the SCA columns, the Field of View layout follows the natural annular curve of an off-axis TMA optimized field. Small non-axial adjustments are used to further optimize the active area of the field. We maintained a $95 \%$ (RMS) polychromatic wavefront error of $<45 \mathrm{~nm}$, half of the $90 \mathrm{~nm}$ budget (based on $1 / 13^{\text {th }}$ wave at $1.2 \mu \mathrm{m}$ ), across the entire field. The optical design residual imaging performance of the Wide Field is shown in Figure 10:

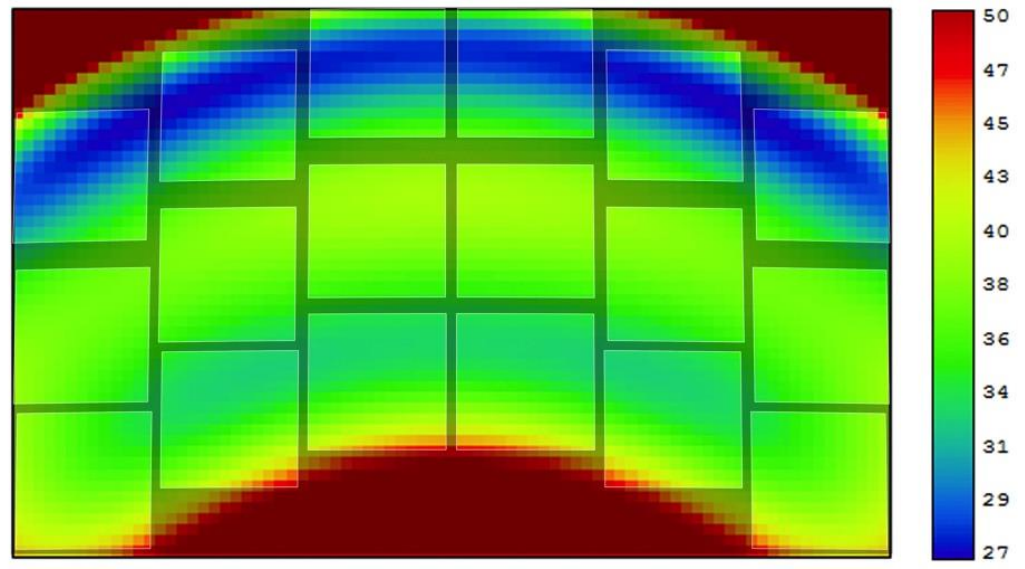

Figure 10. Optical Design Residual Performance of the Wide-Field Imager in RMS WFE (nm) The projected FOV of each sensor (1-18) is overlaid, revealing the effects of distortion. 


\section{WIDEFIELD SPECTROGRAPHIC MODE (WSM) PERFORMANCE}

The grism has been redesigned to optimize various fabrication and I\&T parameters. The performance requirements are looser than the WIM $(<150 \mathrm{~nm}$ RMS), however performance exceeds that requirement over much of the field. (See Figure 11.)
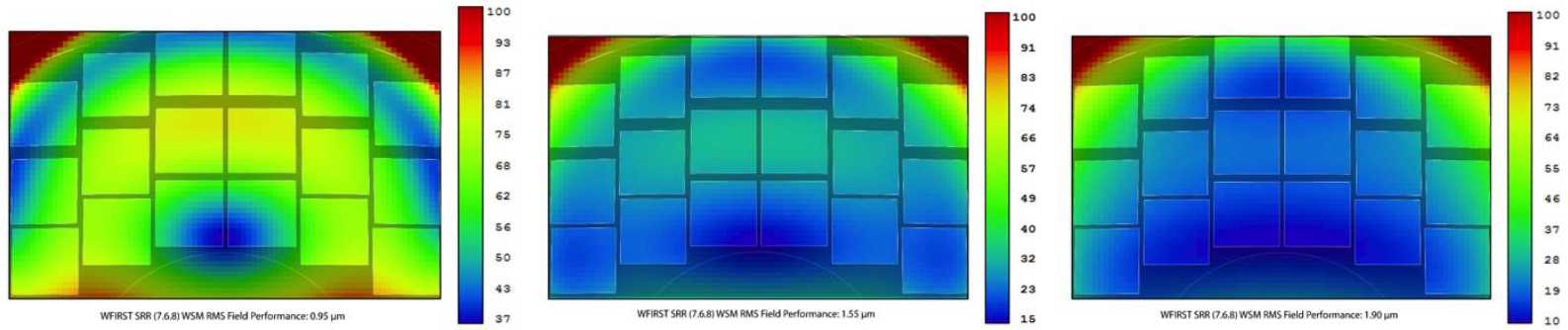

Figure 11. Optical Design Residual Performance of the WSM in RMS WFE (nm) at short, mid, and long wavelengths.

\section{OVERVIEW OF THE INTEGRAL FIELD CHANNEL}

As has been described, within the Wide-Field instrument enclosure is the Integral Field Channel (IFC). The optical bench and Image Slicer detail is shown in Figures 12(a)/(b). The AOM contains the IFC Relay Optics that outputs a collimated beam to the WFI, which is received onto the IFC bench and relayed to the Image slicer before going through the spectrograph.
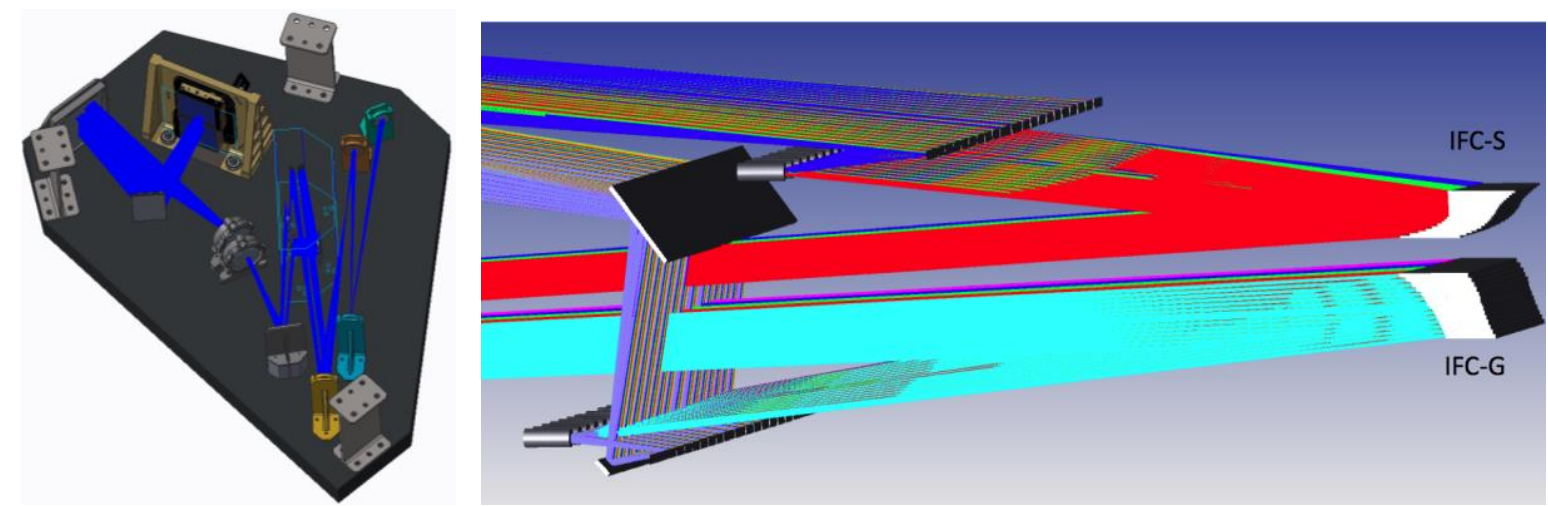

Figure 12. (a) The IFC Optical Bench containing the Receiver, Slicer, and Spectrograph. (b) Optical Layout of the DualField IFC Image Slicer Assembly.

The full IFC optical design, science and performance is discussed in detail in a separate paper in these proceedings. ${ }^{[5]}$

\section{STRAY LIGHT}

The architecture of the wide field Channel allows for an efficient first-order stray light mitigation. To begin, the telescope Outer Barrel Assembly (OBA) will be fitted with internal vanes. The Secondary Mirror sunshade and Primary Mirror central baffle tube work together to prevent direct line of sight "rogue path" light from entering the Aft Optics Module (AOM.) At the telescope intermediate focus there is baffle surrounding the highly caustic curved image. This Entrance Aperture Plate (EAP) is a critical stray light element, as it is the only path for light to pass from the entrance to the exit pupil. By eliminating rogue paths, the pupil and fields are effectively bound. The exit port of the WAO is the Exit Pupil of the entire WFC, thereby cleanly constraining the ray bundles through the entire system.

\section{SUMMARY AND CURRENT EFFORTS}

As we continue to prepare for the System Requirements Review (SRR) in 2017 we are focusing our efforts on the documentation of the optical sensitivities and predictive performance modeling. To anticipate the roadmap to the PhaseB proto-flight design work, we are continuing to evaluate the effects of any inter-disciplinary analyses that would drive 
adjustments to the optical design, though such adjustments are anticipated to be minor. These include mechanical clearances and instrument interface issues as well as thermal, dynamic and integrated modeling results. Scientific simulations will also feedback to our engineering trades. Acquisition strategy decision for various components and subsystems are underway.

\section{CREDITS}

We sincerely acknowledge that this study is the product of a well-coordinated and growing team. This includes the WFIRST-AFTA Formulation Science Working Group, team co-members of many disciplines, contractors, and project leadership and support staff. This work was funded by the National Aeronautics and Space Administration (NASA.)

\section{REFERENCES}

[1] Please refer to the WFIRST Project Page: http://wfirst.gsfc.nasa.gov

[2] Bert Pasquale, David Content et.al, "Optical Design of the WFIRST-AFTA Wide-Field Instrument," in Proc. Of SPIE-OSA International Optical Design Conference, 929305 (2014)

[3] David A. Content, Nerses V. Armani et.al, "Wide field instrument preliminary design for the Wide Field Infra-Red Survey Telescope" in Proc. of SPIE, 88600F

[4] Pasquale, Marx, Content, Kruk, "Widening our View of the Universe", Physics World Optics and Photonics Special Edition, June, 2016, http://live.iop-pp01.agh.sleek.net/2016/05/19/widening-our-view-of-the-universe

[5] Guangjun Gao, Bert Pasquale, et.al, "Optical Design of the WFIRST Phase-A Integral Field Channel," in Proc. Of SPIE-OSA International Optical Design Conference, (2017) 